\title{
Research on Reform and Innovation of Sports Training Mode for Adolescents in China
}

\author{
Jinhua Yuan, Guofeng Deng \\ Nanchang Institute of Science \& Technology, Nanchang City, Jiangxi \\ Province, 330108, China
}

\begin{abstract}
The continuous decline of adolescents' constitution has become a serious social problem. Therefore, the reform of sports training mode for adolescents is expected to improve their physical and healthy levels. In this paper, based on the authors' learning and practical experience, we firstly analyzed the main problems existing in Chinese adolescents' sports training mode, and then put forward the reform and innovation strategies of the adolescents' sports training mode. The research achievements reveal that only by integrating adolescents' sports into the comprehensive reform of education, can adolescents' sports be paid attention to and developed, can the possibility of adolescents' sports participation be realized, and can adolescents' healthy growth be truly promoted.

Keywords: adolescent; sports training; problem; mode; reform; innovation
\end{abstract}

\section{Introduction}

The government has been in charge of the sports cause all the time. The reserve personnel training of large, medium and primary schools and adolescents' amateur sports school is carried out relatively smoothly. However, the system and mechanism that the social forces including training institutions, clubs and private sports schools involved in adolescents' sports training and competitive sports reserve personnel cultivation are not smooth[1]. Therefore, the enterprises and society should participate in the construction of reserve personnel team of adolescents' competitive sports. In this paper, the current situation and existing problems of adolescents' sports training system were in-depth analyzed and 
discussed, and the ideas concerning adolescents' sports training system are put forward, which provides foundation for the decision-making departments to strengthen management and make decision.

\section{Main Existing Problems in Chinese Adolescents' Sports Training Mode}

\subsection{Irrational adolescents' sports reserve personnel structure}

At present, there are less second-line persons in the proportion of reserve personnel in various provinces and cities. The whole structure shows the situation that the base is too big, and the middle part is insufficient. Besides, the "extensive" training mode in the cultivation process also leads to the lowefficiency cultivation. This phenomenon will lead to some problems. On one hand, the number of excellent reserve personnel who joins the first-line team from second-line team is insufficient, which makes the first-line team feel no successors[2]. On the other hand, the pressure of grass-roots training units will increase, which makes the insufficient funds and resources become more deficient, and thus affects the quality of reserve personnel training of third-line sports teams.

\subsection{Lack of the cultural education of athletes training}

The cultural quality of athletes not only directly affects the athletes' understanding of technical movements and coaches' tactical awareness, and the improvement of athletes' competitive level, but also affects the athletes' mental and physical coordinated development, psychological quality improvement and future sustainable development. At present, there are serious deficiencies in the cultivation of athletes' cultural education in the process of training adolescents' sports reserve personnel, whose main reasons are excessive pursuit of competition and the improvement of competitive level, lack of attention to culture and education, and lack of time arrangement for cultural study[3].

\subsection{Loss of athletes and difficulties in reasonable mobility}

As the current adolescents' sports reserve personnel training mainly relies on the national financial allocation or local government investment. Training an excellent athlete in local region will spend a huge cost. Therefore, local protectionism prevails. Various means and even administrative intervention are used to hinder the flow of talented athletes. On the other hand, only a few sports items such as basketball and football conduct professional league, which can be carried out through the operation of the free market transfer. The main flow ways of other items are leasing, exchange, one-time buyout, the normal personnel transfer, co-cultivation, exchange through agreements, contract-signing and directional training. These ways are mainly operated by government functional 
departments, which are easy to produce rent-seeking behaviors that the relevant officials use their power to conduct a variety of black-box operation[4].

\subsection{Insufficient emphasis on athlete human capital property rights}

In the early years, as investment in the adolescents' sports reserve personnel training is entirely conducted by the state, the investment and income subjects are basically clear. With the continuous development of sports cause, more and more investors are also participating in the adolescents' sports reserve personnel training. The relationship of human capital property rights is not clear. Especially, social investment and the investment interests of athletes and their families cannot be guaranteed. The contradictions including the ownership of athletic performance, the development and utilization of intangible assets and the profit of the transfer are outstanding. The interests of all sides are difficult to coordinate, and the corresponding laws and regulations are not set up, which result in the increasing conflicts and disputes.

\section{Reform and Innovation Strategies of Chinese Adolescents' Sports Training Mode}

\subsection{Equilibrium distribution of the number of people in each sports item}

First, reform competition system, and improve the popularization of each sports item for the purpose of reserve talents transfer. The reform of grass-roots amateur training competition should be combined with the actual situation of adolescents' teaching and training. The ranking can be determined based on different proportions of the comprehensive score of special achievements, physical fitness, and basic technology according to the different items and ages, which can avoid not only the unfair phenomenon that the uniformity is imposed in all items, but also the emergence of adultification in adolescents' competition. The promotion of the adolescents' rational choice on sports items attributes to the balanced development of the number of people in each sports item.

Second, the government sports administrative departments should gradually shift the focus of young amateur training to large, medium and primary schools, which expands the number of existing training athletes[5]. The key to speed up the development of competitive sports is to have tens of and thousands of adolescents' competitive reserve personnel. The government should increase the working force of adolescents' amateur training in large, middle and primary schools, and make a good job of basic sports facilities and external environment construction, change the past practices that the government departments are directly in charge of adolescents' amateur training and provide reserve athletes for competitive sports via adolescents' amateur sports schools, and encourage large, medium and primary schools to carry out amateur training via financial subsidies and market guidance mechanism. 


\subsection{Improve the overall strength of the adolescents' reserve personnel mode}

First, the cooperation between professional sports clubs and amateur sports schools, and large, medium and primary schools to enhance their comprehensive strength. In accordance with the requirements of the transformation of government functions under the socialist market economy and the basic principle of "enterprise without superior", the government should revoke investment in professional sports clubs and amateur sports schools, and their property rights are clarified, which makes them truly be the market main body with self-developing and self-financing[6]. On this basis, the government should cooperate with the large, medium and primary schools to organize development and exchange activities of competitive sports talents, and gradually establish economic and technical organizations which carry out training and competition of the excellent athletes and adolescents' amateur training. The measures should also be taken including mutual mobility of athletes, coaches' qualification, the development of training programs and standards and so on. The number of athletes in training should be guaranteed in the process of solving the problem of professional teachers in large, medium and primary schools to achieve a win-win effect.

Second, the preferential policies and measures regarding taxation and management should be formulated, and the domestic and foreign social forces should be encouraged to carry out adolescents' amateur training. The government should withdraw from the general competitive area of sports reserve talents development so that amateur sports schools and professional sports clubs can really enter this area. At present, a large number of private sports schools have emerged in China, which is an important mode for the competitive reserve athletes training of professional athletic clubs. However, their school conditions and the guidance force are uneven. Therefore, in order to improve the quality of all kinds of adolescents' reserve talents training mode, the government should strengthen the management and improvement of the approval, registration and management system of private amateur sports schools, training institutions or clubs to improve the training conditions and quality of the competitive sports reserve talents.

\subsection{Broaden transfer channels of competitive sports reserve talents from the professional sports clubs}

First, the professional sports clubs should be encouraged to cooperate with the primary and secondary schools to cultivate competitive sports reserve talents. However, in regards to the actual situation in Sichuan province, professional sports just take a solid footsteps, and there are still many problems in professional sports clubs, whose funds cannot rely entirely on their own. It is not realistic that each club establishes a few adolescents' amateur training team by completely copying the experience of other provinces. Therefore, the cooperation between the clubs and primary and secondary schools in adjacent areas should be further promoted. The clubs provide funds and professional technical guidance. The primary and secondary schools are responsible for cultural education and daily 
management. Adolescents' competitive sports reserve talents are cultivated by their mutual cooperation. The clubs has the right of first choice of the cultivated talents.

Second, the "paid delivery" mechanism of transferring competitive sports reserve talents to the elite sports team should be established. Shortage of funds has become a common problem in various amateur training units. At present, it is not enough to only rely on the limited capital investment of the state. The funds channels for amateur training should be broadened via seizing the opportunity, relying on the society, and boldly starting the reform from amateur training system[7]. According to the principle of "who invests, who benefits" which adapts to the market economic system, the reform made must meet the requirements of the market economy. A sound legal system for the movement of adolescents' competitive sports reserve talents should be established. These legal rules and regulations can be used to conduct standard management of the movement of adolescents' competitive sports reserve talents to a variety of professional and amateur sports organizations, which can make the cultivator get appropriate rewards, break the local protection, curb the bad competition, and meet the supplement of competitive sports reserve talents.

\section{Conclusion}

Sports intervention is an effective means to enhance the physical health of adolescents. In regards to the current social environment of the development of adolescents' sports, the implementation of comprehensive reform of adolescents' sports is a rational choice to promote the participation of adolescents' people in sports. Comprehensive reform of adolescents' sports should be integrated into the educational comprehensive reform, and be combined with the education system reform. The schools, society and families should provide more opportunities for adolescents to participate in sports, and make them develop good habits of physical exercise, which can fundamentally promote the growth of adolescents with the physical and mental health.

\section{References}

[1] LUO Huaping. Research on the teaching mode of adolescents' sports club. Adolescents Sports, (07), pp.10-11, 2016.

[2] QIU Ting, JIANG Han, LIU Mingyi. Research on Chinese adolescents' sports competition reform path. Sports Culture Guide, (08), pp.5-8, 2014.

[3] ZHANG Chuoan. Rational thinking on adolescents' sports comprehensive reform. Journal of Beijing Sport University, (08), pp.1-5, 2014.

[4] Pilot effect of Zhejiang adolescents' sports club reform. Adolescents Sports, (04), pp.7-9, 2014. 
[5] GUO Jianjun. Adolescents' sports work report. Adolescents Sports, (03), pp.1$5,2014$.

[6] ZHANG Chuoan. On the interests of adolescents' sports comprehensive reform. Journal of Hebei Institute of Physical Education, (02), pp.1-3, 2014.

[7] WANG Bin, WU Baozhan, LI Xia. Research on the reform and innovation of adolescents' sports training system in Sichuan Pprovince. Sports World (Academic Edition), (12), pp.23-25, 2012. 